\title{
On the Treatment of Ulcerative Colitis with Humid and Heat Accumulation Based on Tri-Jiao Dialectics
}

\author{
Xia Xiao", ${ }^{1,}$ Yaping Cui ${ }^{2}$, Zihua Guo ${ }^{1}$, Chuanchuan Bi $^{1}$, Qiangqiang Gao $^{2}$ \\ ${ }^{1}$ Shaanxi University of Traditional Chinese Medicine, Xianyang, Shaanxi 712046, China. \\ ${ }^{2}$ Affiliated Hospital of Shaanxi University of Traditional Chinese Medicine, Xianyang, Shaanxi 712000, China.
}

How to cite this paper: Xia Xiao, Yaping Cui, Zihua Guo, Chuanchuan Bi, Qiangqiang Gao. (2020) On the Treatment of Ulcerative Colitis with Humid and Heat Accumulation Based on Tri-Jiao Dialectics. International Journal of Clinical and Experimental Medicine Research, 4(4), 225-227.

DOI: $10.26855 /$ ijcemr.2020.10.017

Received: September 29, 2020

Accepted: October 22, 2020

Published: November 9, 2020

*Corresponding author: Xia Xiao, Shaanxi University of Traditional Chinese Medicine, Xianyang, Shaanxi 712046, China.

Email: 1124078307@qq.com

\begin{abstract}
Ulcerative colitis is one of the common refractory diseases in gastroenterology. The course of ulcerative colitis is prolonged. Modern medicine is mainly treated with anti-inflammatory and other symptomatic support, but the results are not satisfactory. In recent years, Chinese medicine has a remarkable curative effect in the treatment of such diseases, and the treatment of traditional Chinese medicine has the characteristics of "simple, effective, cheap and excellent" characteristics. Based on the three-coke dialectics, combined with the pathological characteristics of modern ulcerative colitis patients, this paper thinks that the occurrence and development of ulcerative colitis is closely related to the three-coke. The three-coke treatment runs through the treatment of ulcerative colitis and provides a new idea for the treatment of ulcerative colitis.
\end{abstract}

\section{Keywords}

Tri-Jiao Dialectics, Ulcerative Colitis, Tri-Jiao Treatment

Ulcerative colitis (ulcerative colitis, UC) is a chronic non-specific inflammatory bowel disease. Its lesion site is mostly in rectum and sigmoid colon. In severe cases, it can involve the whole colon. The etiology and pathogenesis of ulcerative colitis are not completely clear. It is generally believed that it may be related to the comprehensive role of environmental, immune, genetic, intestinal flora disorder and other factors [1]. As the main clinical manifestation of this disease, abdominal pain, diarrhea, purulent blood, acute and severe, the course of disease is generally longer, the severity of the disease is different, easy to relapse after the disease, according to the disease can be divided into active and remission period, in which acute onset, severe symptoms, and systemic symptoms are obvious, is recognized as one of the [2] of modern refractory diseases in the world. At present, western medicine can control the disease to a certain extent, but there are still many side effects, easy to relapse after the disease, long-term use easy to produce drug resistance and many other problems [3].

Chinese medicine will ulcerative colitis as "dysentery”, "diarrhea”, "intestinal fetishism”, "blood stool”, and so on, the cause of its mechanism, "Su-Quan really want to big theory": "Shaoyang Si Tian, fire to win, people's disease release red white." "The young and the old are in the spring, and the fire is the best, and the people's diseases are filled with red and white, and the abdominal pain is red." "Danxi heart method dysentery": "red dysentery is from the small intestine, white dysentery from the large intestine, are damp-heat-based". The treatment of ulcerative 
colitis caused by later generations of doctors from the gastrointestinal "wet, heat, fire, poison" syndrome differentiation treatment, when the disease is more serious, is not appropriate. If the condition is prolonged, it can cause dehydration and even endanger the patient's life. From the clinical practice, the author found that the treatment of dampness and heat accumulation ulcerative colitis combined with Sanjiao Dialectics has a good effect, and now the treatment ideas are arranged as follows:

\section{Relationship between Sanjiao and damp-heat ulcerative colitis}

The concept of "Three Jiao" originated from "Huangdi Neijing" said: "gallbladder, stomach, large intestine, small intestine, bladder, three Jiao six Fu organs are all Yang” ("Su-Quan Jin Gui Zhen speech"), is the "five viscera six Fu organs". Zhang Zhongjing of the Eastern Han Dynasty ("Treatise on typhoid fever and miscellaneous diseases") gave the "three Jiao Dialectics" to the "six Classics Dialectics", such as "typhoid fever on the differentiation of Yangming disease pulse syndrome and treatment of the eighth": "eating grain to vomit, belong to Yangming also, Evodia decoction master. Soup against the drama, belong to the Jiao also [4]", or "woman typhoid fever, fever, water, clear day, ... No stomach qi, and the upper two coke, will heal itself”. Tang Sun Simiao ("Preparation of Qianjin Yaofang”) elaborated the position and concept of Sanjiao, and put forward the treatment and prescription of Sanjiao cold and heat deficiency. To the Qing Dynasty Wu Jutong ("febrile disease differentiation"), create three-Jiao dialectical and emphasize the location of the viscera, said that the upper-Jiao heart and lung, middle-Jiao spleen and stomach, lower-Jiao liver and kidney, later generations of doctors have benefited a lot.

The three-coke is divided into upper, middle and lower coke, which corresponds to the corresponding viscera part of the human body, thus corresponding to the corresponding function. For example, "the upper coke is like fog", "the middle coke is like blasphemy", "the lower coke is like blasphemy", from the distribution and function relation of the three coke, it can be seen that the most closely related damp-heat enteritis belongs to the lower coke, but cannot be separated from the upper, middle and lower coke [5] from the whole treatment. The upper coke of the lung is the upper source of water, and because of the appearance of the lung and the large intestine, the transmission relationship between the lung qi and the large intestine is very close. Lung main gasification, can be scattered water valley fine, Qi and blood body fluid, gasification is wet nowhere to hide. Spleen in the middle coke, transport water wet, the ancients called it the middle soil with the four irrigation, if the spleen lost health, water and liquid distribution lost, it is also wet, accumulated dampness and heat for a long time, dampness and heat wrapped, difficult to decompose. Wet sticky swim down, but also wrapped in evil heat, obstruction of large intestine. Also because of the time more fat sweet spicy food, can also brew the evil of dampness and heat, thus aggravating spleen deficiency. Intestinal Fu has been evil, coupled with the dampness and heat of the middle coke, Sanjiao and pathogenic, qi and blood Yong stagnation, fat collateral injury.

Dampness and heat type is the most common [6] of TCM syndrome differentiation in ulcerative colitis. Wet for Yin evil, its nature heavy turbid sticky, damp-heat phase sum, wet accumulation heat Sheng, hot steam wet movement, diffuse three coke. Heat into the wet, such as oil into the surface, is difficult to decompose. Dampness and heat depend on each other, latent body, difficult to quickly treat, the disease is difficult to heal for a long time, then become "dampness and heat evil"; dampness is very Yang micro, heat Sheng is boil Yin, deficiency of qi, exorcism is more powerless.

\section{Treatment of Ulcerative Colitis from Trijiao}

\subsection{Replenish lung qi and promote qi}

Lung is Yin, Jiao dirty, ulcerative colitis for chronic disease, long-term disease can consume gas injury Yin, so damage to the lung. At the same time, the main body of lung qi, lung qi impassable affects the operation of the whole body Qi machine, thus affecting the distribution of semen, and then affects the function of the intestine. Therefore, the intestinal tract can be added to nourish the lung and nourish yin, such as Radix Pseudostellariae, Codonopsis, Astragalus, etc.

Large intestine as the conduction of the official, to pass for the smooth, to drop for the sum. Patients with ulceration can be seen under the benefit, acute after heavy, even mucus purulent blood stool, this is large intestine function loss. The motive force of large intestine operation comes from qi, so the qi machine follows the large intestine, so the method of promoting lung and regulating qi can help the large intestine to conduct dross function normally. As the "Renzhai straight refers to the square" cloud: "out of the accumulation of stagnation, no accumulation cannot be". It can be seen that the method of promoting lung and regulating qi is particularly important in the treatment of ulceration. Therefore, in the treatment can choose Chen Pei, Platycodon grandiflorum and other Qi products. In 
addition, Qi stagnation can cause blood stasis for a long time, so long-term disease can see qi and blood stasis, disease into the blood points, symptoms see the lower purulent blood stool. Therefore, the treatment can be added to "stop bleeding not left, not injured” products, such as: Panax notoginseng, Puhuang, can make blood stasis.

\subsection{Replenish spleen and stomach, remove dampness and heat}

Although the etiology is complex, it is mostly related to spleen deficiency, dampness and heat. Spleen deficiency is the premise and foundation of dampness and heat retention, and it is also the inducing factor of dampness and heat evil qi surging. Therefore, in the clinical use of dampness and heat products can be added to protect the spleen and stomach, such as: yam, Atractylodes macrocephala, Poria cocos, licorice and so on.

The initial and acute stage of the disease is mostly damp-heat accumulation of large intestine, treatment should be to clear heat and dampness as the method, can be added or subtracted mannose disinfection Dan.

\section{Examples of medical records}

The patient was 46 years old, first diagnosed on December 4, 2019. Colonoscopy before February, Chronic ulcerative colitis, Oral and enema Chinese and western medicine treatment is ineffective, In recent days, these symptoms are aggravated by eating uncleanly, Present symptom: stool is thin, Three to four times a day, There's blood and mucus, In a hurry and heavy, Fatigue, Nathan, Red tongue, yellow fur, The veins are smooth. Fecal routine: pus cells (++), white blood cells (+). The patient's course is long, the symptoms are mixed with reality, its pathogenesis is spleen and stomach weakness, dampness and heat, to clear heat and dampness, and invigorate spleen and invigorate qi, reconcile qi and blood. Prescription: Yinchen $30 \mathrm{~g}$ talc $15 \mathrm{~g}$ Rhizoma Coptidis $15 \mathrm{~g}$ Acorus calamus $20 \mathrm{~g}$ White nutmeg 6 g patchouli 10, Astragalus $30 \mathrm{~g}$ yam $20 \mathrm{~g}$ Angelica sinensis 15, licorice $5 \mathrm{~g} 7$.

Second diagnosis (December 12): stool is not formed with mucus, 2 times a day, no pus.

Blood, still feel weak, anorexia, tongue red, fur slightly yellow greasy, thin veins. Top talc, patchouli plus Amomum (lower)5 g Hawthorn $10 \mathrm{~g}$ Shenqu $10 \mathrm{~g} 10$.

Third diagnosis (December 23): stool without mucus pus blood, appetite can, tongue pale red, fur white greasy, thin veins. Fecal routine: no pus cells and white blood cells were found. Six gentleman decoction to reduce spleen qi, even take January. The colonoscopy showed that there was no obvious abnormality in the colon.

\section{Conclusion}

Collapse is a chronic persistent disease, its pathogenesis is still unclear, various treatments are not satisfactory. This disease belongs to the syndrome of deficiency and deficiency in traditional Chinese medicine. The clinical manifestation is complex. Besides gastrointestinal manifestations, there are skin and lung manifestations. Traditional Chinese medicine has been treating this kind of disease for a long time, and the curative effect is remarkable. The major doctors have rich clinical experience for the reference of later generations.

Ulcerative colitis is closely related to the pathogenesis and development of the disease, and can be treated by Sanjiao.

\section{References}

[1] Ni Liangyu. (n.d.). Dialectical treatment of chronic epididymitis [J]. and Jilin traditional Chinese Medicine, $28(1): 16$.

[2] Huang Xiangyang, Bao Yanzhong, Qiu Xinying. (n.d.). 175 [J]. of chronic epididymitis treated by adding or subtracting xiangqi powder. Journal of Zhejiang Institute of traditional Chinese Medicine, 25(6): 31.

[3] Zhao Yupei. (2015). Chen Xiaoping. Surgery [M]. Version 3. Beijing: People’s Health Publishing House, $2015: 732$.

[4] Han Zhang Zhongjing. (2007). [M]. On typhoid fever Beijing: Xueyuan Publishing House.

[5] Liu Yuning, Fang beloved, Chen Yiping. (2016). Ideas and Methods of Dialectical Treatment of Three Charges in Kidney Disease [J]. Chinese Medicine Chinese Journal of Integrated Chinese and Western Medicine Nephropathy, 2016, 17(5): $377-380$.

[6] Jiang Liqin. (2014). Effect of invigorating spleen and clearing heat and removing wet method on the treatment of slow release of ulcerative colitis with spleen deficiency and dampness and heat [D]. Nanjing University of Traditional Chinese Medicine. 